\title{
Mixed Simulation Approach For Direct Connection Between Power Amplifiers And Antenna Arrays Without The Use Of Isolators
}

\author{
G. Zakka El Nashef, F. Torrès, S. Mons, T. Reveyrand, E. Ngoya, M. Thévenot, T. Monédière, R. Quéré
}

XLIM - C2S2/OSA departments UMR CNRS n ${ }^{\circ} 6172$, University of Limoges 123, avenue Albert Thomas 87060 Limoges CEDEX Tel: +33 555457520

\begin{abstract}
This paper describes a mixed simulation approach of electromagnetic (EM) macromodel and nonlinear circuit bilateral model to correct the output signal for a power amplifier (PA) and obtain an optimal radiation pattern for an active antenna array transmit (TX) chain. Various experimental results show the reliability of EM, nonlinear PA models and mixed simulation approach as well. The originality of this work is the elimination of isolators between PAs and antennas while maintaining a matched PA and a correct radiation pattern in regards to the antenna's high impedance mismatch.
\end{abstract}

Index Terms - Active antenna, behavioral models, cosimulation, electromagnetic modeling, power amplifiers.

\section{INTRODUCTION}

It is now well known that the mutual coupling between elementary antennas of a steered active array significantly impacts the input impedance of these antennas. Moreover, this mutual coupling is affected by the pointing direction of the array, thus leading to the variation of the impedances of the antennas. Therefore, most of today's active antennas arrays include isolators between the PAs and the antennas. The isolator plays an important role to minimize the PA load variations and preserve its linearity. It prevents the transistor from saturating by dissipating the reflected power in a load resistor which terminates the isolator's third port. Unfortunately, isolators are bulky and expensive devices and do not address the future requirements for low cost and size reduction, which likely involve eliminating isolators. But if isolators are to be avoided, PAs will be directly connected to antennas, and as the antenna's impedance varies (with frequency or pointing angle), the mismatching will clearly affect the PAs performances in terms of gain (AM/AM) and phase (AM/PM), thus modifying the necessary steering weights applied to the array and degrading its efficiency and radiation performance. Therefore, a mixed simulation approach involving EM models for the antenna and behavioral bilateral PA models is needed to take into account both effects (EM and nonlinear) in order to correctly handle the direct interaction between PAs and antennas.

In this paper, PA and EM models are discussed in section II. A simple combination of both models, called "mixed simulation approach", was developed using ADS (Advanced Design System) from Agilent, and will be discussed in section II-C. Finally, an active demonstrator was realized and measured, without the use of isolator between PA and antenna, to validate the concept of the mixed simulation approach for a direct bilateral communication between PA and antenna.

\section{OVERVIEW OF THE MIXED SIMULATION APPROACH}

The development of rigorous nonlinear and EM models may thus be of valuable help for establishing the accuracy of system level simulation. In this section, we show that nonlinear and EM models can be effectively combined to produce an efficient mixed simulation approach, which allows us to accurately model the behavior of the PA in presence of a mismatched antenna without the use of isolators. Hence, the obtained simulated data could describe adequately the mismatching in function of passive (antennas) and active (PA) elements and, in principle could be used for real-time correction in order to optimize performances of the global system.

\section{A. Power Amplifier behavioral model}

The direct connection between PA and antennas has severe consequences on PA performance, e.g. a deviation from the optimum load impedance can reduce the maximum power delivered to the antenna and change considerably the PA linearity. It may lead to instability or breakdown of the PA output stage due to high VSWR, making the mismatching effect a major challenge and that must be taken into account. Therefore, a PA bilateral behavioral model, based on nonlinear scattering functions [1], was developed. This model takes into account strong output loading impedance mismatch, i.e. Voltage Standing Wave Ratio (VSWR) up to four, and neglects the memory effects. Extensive mathematical development can be found in [1].

Model identification and nonlinear scattering functions extraction are based on load-pull characterization [2] of microwave PA, allowing us to acquire complete and accurate information of PA electrical behavior, which in turn enables us to define and validate a nonlinear behavioral model.

In order to validate the model, we used ADS to implement it, using three main blocks: HB (Harmonic Balance) simulation block, FDD (Frequency-domain Defined Device) nonlinear block and DAC (Data Access Component) block. These blocks will allow us to solve nonlinear equations on one hand, and to interpolate extracted large signal S-parameters (measurement file) on the other hand. The model validation is shown later in section II-C. 


\section{B. Electromagnetic macromodel}

For the EM part, a synthesis macromodel, based on [3], was developed to determine the mismatching impedances $(\neq 50 \Omega)$ and calculate the necessary steering weights for each element of the array according to the frequency and the pointing angle. This technique takes into account the mutual coupling between array elements (antennas) while maintaining a zero reflection coefficient, i.e. isolators become useless, and an optimal radiation pattern is obtained (high gain and low side lobes levels). Some highlights are needed before describing the prototype and discussing the measured results.

The direct connection between the antenna and PA and the strong mutual coupling between different elements of the array affect the antenna input impedance, leading to a strong mismatching problem which will modify the performance of the array in term of radiation pattern: the array efficiency and its radiation performance will be degraded. Therefore, we developed an EM macromodel, based on [3], in order to calculate, for each array element, the matching impedance $\left(Z_{\text {match }} \neq 50 \Omega\right)$ as well as the feeding weights (magnitude and phase), according to pointing angle and frequency. We should also note that the optimized matching impedances of antennas are different from the ideal $50 \Omega$ value, showing the important influence of mutual coupling and the need to take this phenomenon into consideration. Thus, to realize the overall matching of the array and limit the effect of mutual coupling on the antenna access $\left(Z_{\text {match }} \neq 50 \Omega\right)$, the antennas must be fed with the calculated power input waves " $a$ " through ports having the input impedances that are conjugates of the matching impedances, $Z_{E i}=Z_{\text {match }}{ }^{*}$. This means that, for a given pointing angle, feeding each antenna with the correct steering weights (amplitude and phase) calculated with the EM model automatically ensures a correct matching of each port, while achieving an optimal radiation pattern. This particular point allows maintaining a zero reflection coefficient, thus leading to the uselessness of isolators. Complete mathematical developments and details can be found in [3].

In this paper, an experimental prototype working at $8.2 \mathrm{GHz}$ was realized to validate the synthesis EM macromodel (Fig. 1). It consists of a linear 8-element array of patch antennas, whose inter-element distance has been reduced to $0.4 \lambda_{0}$ to increase mutual coupling between antennas, and variable attenuators and phase shifters to manually set the steering weights of the array. A full-wave simulation of the microstrip array is performed with CST MWS, each antenna being fed by the optimal power wave $a_{i}$ and the matching impedances $Z_{\text {match }}$, which simultaneously satisfy the objective radiation pattern and the matching of all the feeding ports. The simulated radiation patterns for $-20^{\circ}$ and $+30^{\circ}$ pointing angle obtained by linear combination using CST MWS, are compared to the measured radiation patterns (Fig. 5 and Fig. 6) with excellent results, despite of a large VSWR (up to 3 for certain antennas).

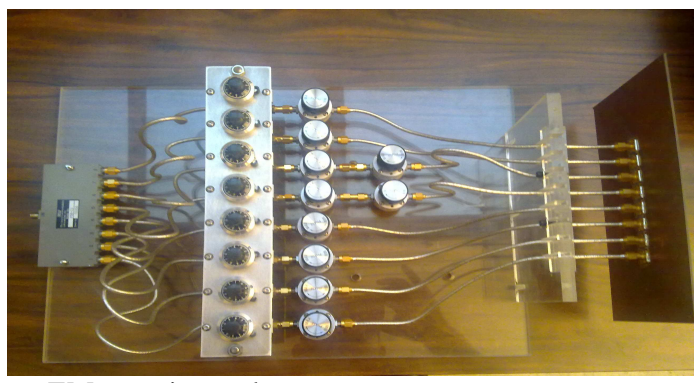

Fig.1. EM experimental prototype

\section{Mixed simulation approach}

In order to observe the interaction between the antenna and the PA, the idea of mixed simulation approach was to use the calculated matching impedances obtained from the EM macromodel as load impedances for the PA: load-pull measurements are performed on the PA loaded with a wide range of impedances (corresponding to a VSWR spanning from 1 to 4) controlled with an automated tuner [2]. These impedances must include the matching impedances calculated by the EM model. Model parameters are extracted from these measurements, and implemented in Agilent's ADS.

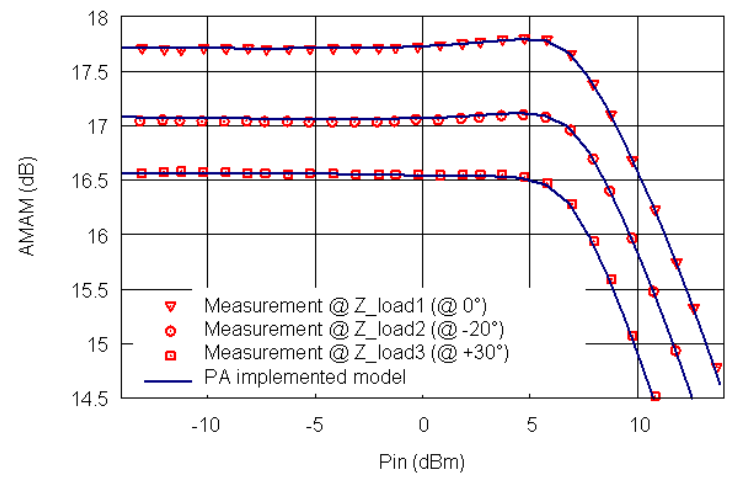

Fig.2. AMAM vs. Input power for calculated EM model impedances $\left(Z \_l o a d 1=46.1+\mathrm{j} \cdot 9.7, \mathrm{Z} \_\right.$load $2=42.3+\mathrm{j} \cdot 21.5, \mathrm{Z} \_$load3 $=25.5+\mathrm{j} \cdot 18.5$ ). Model (lines) compared to load-pull measurement (symbols). $\mathrm{F}_{0}=8.2 \mathrm{GHz}$

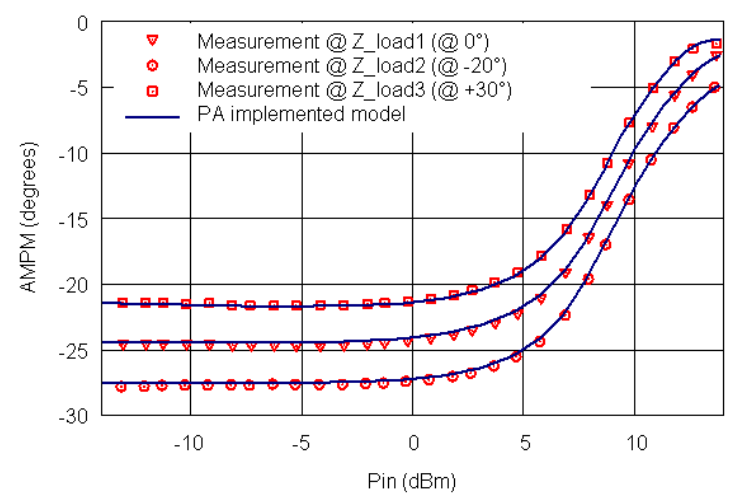

Fig.3. AMPM vs. Input power for calculated EM model impedances $\left(Z \_l o a d 1=46.1+\mathrm{j} \cdot 9.7, \mathrm{Z} \_\right.$load $2=42.3+\mathrm{j} \cdot 21.5, \mathrm{Z} \_$load 3 $=25.5+\mathrm{j} \cdot 18.5$ ). Model (lines) compared to load-pull measurement (symbols). $\mathrm{F}_{0}=8.2 \mathrm{GHz}$ 
Fig. 2 and Fig. 3 show the comparison between the ADSimplemented model (Section II-A) and the measurements in terms of gain (AMAM) and phase (AMPM) for three calculated load impedances that correspond respectively to pointing angles of $0^{\circ},-20^{\circ}$ and $+30^{\circ}$. Fig. 2 and Fig. 3 show a perfect agreement, which clearly demonstrate the efficiency and accuracy of the PA behavioral model, even in the presence of the actual loading impedances of the antennas.

\section{ACTIVE ANTENNA DEMONSTRATOR}

To demonstrate the effectiveness of the proposed mixed simulation approach and the feasibility of a TX chain without isolators while maintaining an optimal radiation pattern and matching of the PA. An active antenna demonstrator was realized, based on the one presented in Fig. 1, with the addition of eight PAs directly connected to antennas (Fig. 4).

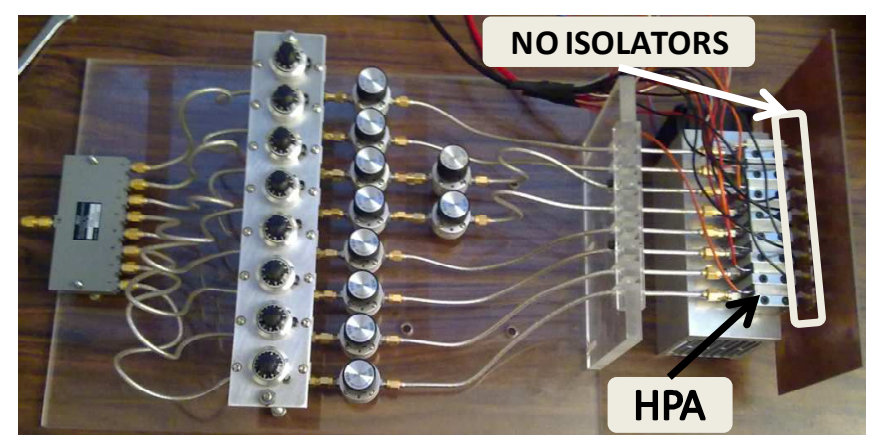

Fig.4. Active antenna demonstrator

The exact steering weights needed to achieve an optimal radiation pattern and a null reflected wave from each antenna of the array are calculated from the EM model, along with the antennas input impedances. The amplitude (AMAM) and phase (AMPM) modifications due to the PAs feeding such impedances are given by the PA model. Then, the steering weights to apply to each PA's input have to be modified to account for the modifications due to the PAs. These final values are then set through the variable attenuators and phase shifters. The desired radiation pattern is then obtained and the matching conditions are fulfilled, thus making the isolator useless.

Fig. 5 and Fig. 6 compare the measured radiation patterns with PA (active antenna demonstrator), the measured radiation patterns without PA (EM macro-model validation), and simulated radiation patterns without PA (CST) for $-20^{\circ}$ and $+30^{\circ}$ pointing angles. These results show a good agreement between the main lobes for the measured results (with and without PA) and the simulated one. Some slight differences on the side lobe levels can be noticed, which have been related to some imperfections in the measurement setup.

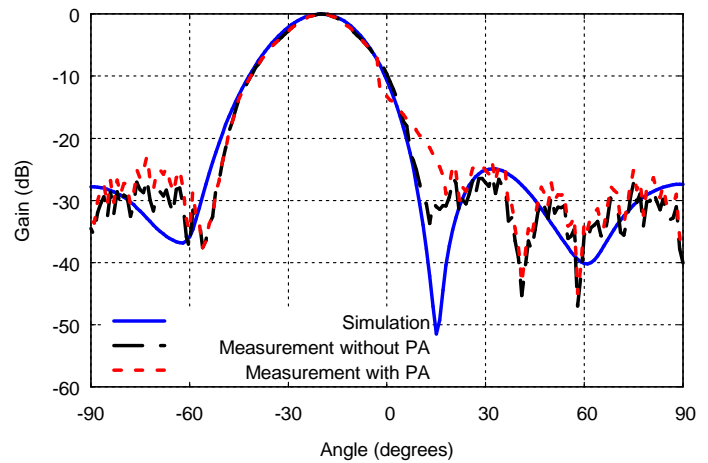

Fig. 5. Radiation pattern comparison $\left(-20^{\circ}\right)$. Active demonstrator measurement (solid line). Measurement without PA (big dashed line). EM macro-model simulation (small dashed line). $\mathrm{F}_{0}=8.2 \mathrm{GHz}$.

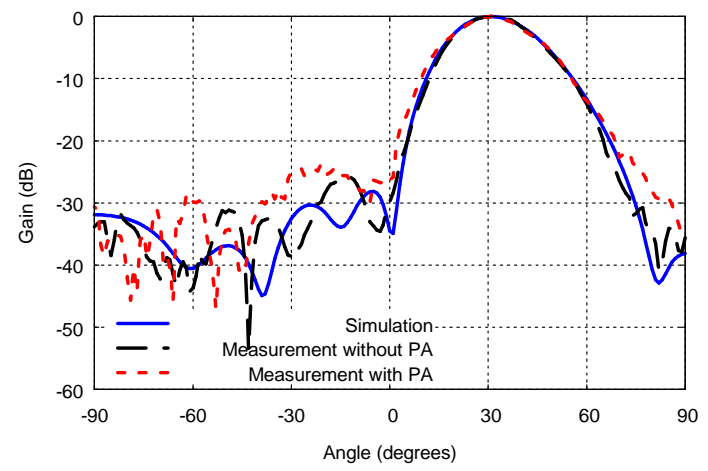

Fig. 6. Radiation pattern comparison $\left(+30^{\circ}\right)$. Active demonstrator measurement (solid line). Measurement without PA (big dashed line). EM macro-model simulation (small dashed line). $\mathrm{F}_{0}=8.2 \mathrm{GHz}$.

\section{CONCLUSION}

We have presented in this paper an experimental validation of mixed simulation approach combining a PA behavioral model which has shown accurate prediction ability for the PA performances even with the presence of high VSWR (up to four), and an EM macromodel taking antennas coupling into account while allowing array synthesis. The combined use of these models led to correct radiation patterns with a direct connection between PAs and antennas, i.e. the elimination of isolators.

\section{REFERENCES}

[1] J. Verspecht, "scattering functions for nonlinear behavioral modeling in the frequency domain," Fundamentals of Nonlinear Behavioral Modeling, Foundations and Applications Workshop, IEEE MTT-S International Microwave Symposium, June 2003.

[2] T. Reveyrand, T. Gasseling, D. Barataud, S. Mons, J-M. Nébus, "A Smart Load-Pull Method to Safely Reach Optimal Matching Impedances of Power Transistors," IEEE MTT-S Digest, Honolulu, Hawaii, June 2007, pp.1489-1492.

[3] A.E.S. Ahmad, M. Thevenot, C. Menudier, M. Koubeissi, E. Arnaud, T. Monediere, "Design of a coupled antenna array for mobile HyperLAN2 applications," accepted for publication in International Journal of Microwave and Wireless Technologies, 2011. 